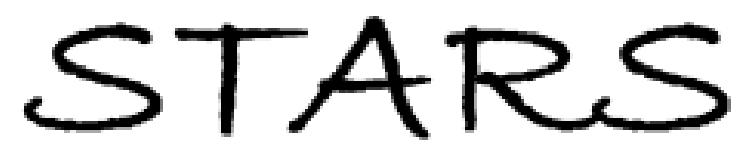

University of Central Florida

STARS

$1-1-2007$

\title{
Modified Beckmann-Kirchhoff scattering model for rough surfaces with large incident and scattering angles
}

James E. Harvey

University of Central Florida

Andrey Krywonos

University of Central Florida

Cynthia L. Vernold

University of Central Florida

Find similar works at: https://stars.library.ucf.edu/facultybib2000

University of Central Florida Libraries http://library.ucf.edu

This Article is brought to you for free and open access by the Faculty Bibliography at STARS. It has been accepted for inclusion in Faculty Bibliography 2000s by an authorized administrator of STARS. For more information, please contact STARS@ucf.edu.

\section{Recommended Citation}

Harvey, James E.; Krywonos, Andrey; and Vernold, Cynthia L., "Modified Beckmann-Kirchhoff scattering model for rough surfaces with large incident and scattering angles" (2007). Faculty Bibliography 2000s. 7216.

https://stars.library.ucf.edu/facultybib2000/7216

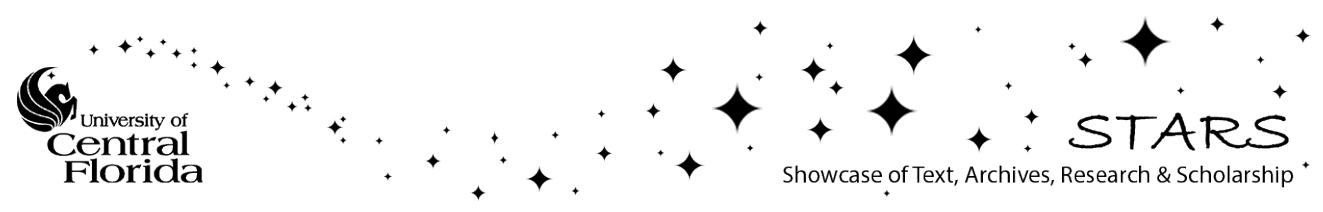




\section{Modified Beckmann-Kirchhoff scattering model for rough surfaces with large incident and scattering angles}

\author{
James E. Harvey, FELLOW SPIE \\ Andrey Krywonos \\ Cynthia L. Vernold \\ The University of Central Florida \\ The Center for Research and Education \\ in Optics and Lasers (CREOL) \\ P.O. Box 162700 \\ 4000 Central Florida Boulevard \\ Orlando, Florida 32816 \\ E-mail: harvey@creol.ucf.edu
}

\begin{abstract}
Surface scattering effects are merely diffraction phenomena resulting from random phase variations induced on the reflected wavefront by microtopographic surface features. The Rayleigh-Rice and Beckmann-Kirchhoff theories are commonly used to predict surface scattering behavior. However, the Rayleigh-Rice vector perturbation theory is limited to smooth surfaces, and the classical Beckmann-Kirchhoff theory contains a paraxial assumption that confines its applicability to small incident and scattering angles. The recent development of a linear systems formulation of nonparaxial scalar diffraction phenomena, indicating that diffracted radiance is a fundamental quantity predicted by scalar diffraction theory, has led to a reexamination of the classical BeckmannKirchhoff scattering theory. We demonstrate an empirically modified Beckmann-Kirchhoff scattering model that accurately predicts nonintuitive experimental scattering data for rough surfaces at large incident and large scattering angles, yet also agrees with Rayleigh-Rice predictions within their domain of applicability for smooth surfaces. () 2007 Society of Photo-Optical Instrumentation Engineers. [DOI: 10.1117/1.2752180]
\end{abstract}

Subject terms: Nonparaxial surface scattering; Beckmann-Kirchhoff surface scattering theory.

Paper 060692R received Sep. 29, 2006; revised manuscript received Jan. 5, 2007; accepted for publication Jan. 8, 2007; published online Jul. 2, 2007. This paper is a revision of a paper presented at the SPIE conference on Scattering and Surface Roughness II, July 1998, San Diego, Calif. The paper presented there appears (unrefereed) in SPIE Proceedings Vol. 3426.

\section{Historical Background of Surface Scattering Theory}

One of the earliest investigators of scattering from a rough surface was Lord Rayleigh. In 1896 he was investigating the reflection of acoustic waves. ${ }^{1}$ Later he noted the effects of poorly polished surfaces on optical performance and examined the effects of surface roughness and angle of incidence on the reflected beam. ${ }^{2}$ In 1907 Lord Rayleigh published an extensive vector perturbation theory of scattering from periodically corrugated reflection gratings, which was an extension of his previous work on the theory of sound. ${ }^{3}$ In 1919 Chenmoganadam ${ }^{4}$ derived a theory of scattered light based on the phase shift of the reflected beam due to the rough surface. Fano ${ }^{5}$ (1941) expanded the Rayleigh approach to explain anomalous diffraction gratings and quasistationary waves on metallic surfaces. However, it was not until World War II, when the problem of background clutter in radar applications became apparent, that a determined effort was made to solve the scattering problem for random surfaces. It was this problem of radar scattering from the sea, associated with the detection of naval targets, that motivated the work of Rice ${ }^{6}(1951)$, and others. ${ }^{7-10}$ Considerable work was also done during the 1960s in attempting to explain radar reflection from the moon. ${ }^{11-14}$

Surface scattering effects can also be described as

0091-3286/2007/\$25.00 @ 2007 SPIE merely diffraction phenomena resulting from random phase variations induced on the reflected wavefront by microtopographic surface features. The Kirchhoff approximation was first introduced in 1952 by Brekhovskikh ${ }^{15,16}$ and applied by Isakovich ${ }^{17}$ (1952) to statistically rough surfaces. It was later treated in English by Eckart $^{18}$ (1953) and Davies $^{19}$ (1954); however, it is the monograph by Beckmann and Spizzichino ${ }^{20}$ (1963) that has become the common reference in the western world.

These two historical approaches, the Rayleigh-Rice ${ }^{6}$ and the Beckmann-Kirchhoff ${ }^{20}$ theories, are commonly used to predict surface scattering behavior. The Rayleigh-Rice vector perturbation theory agrees well with experimental wideangle measurements of scattering from smooth $\left(\sigma_{s} / \lambda \ll 1\right)$ surfaces for arbitrary incident and scattering angles. However, not all applications of interest satisfy the smoothsurface approximation. The Beckmann-Kirchhoff scattering theory is valid for rougher surfaces, but contains a paraxial (small-angle) assumption that limits its ability to accurately handle wide-angle scattering and large angles of incidence. Church et al. published a myriad of papers (starting in about 1975) discussing applications of the Rayleigh-Rice theory in the applied optics community. ${ }^{21-25}$ Elson and Bennett (1979) published a similar perturbation approach to optical surface scattering that proved to yield identical expressions. ${ }^{26}$ Bennett has devoted much of her career to the characterization of scattering surfaces, ${ }^{27,28}$ and Stover 
has specialized in the measurement and analysis of surface scattering data. ${ }^{29,30}$

In 1970, Nicodemus introduced the four-dimensional bidirectional reflectance distribution function (BRDF), defined as reflected radiance divided by incident irradiance, in an attempt to (geometrically) characterize the scattering properties of a surface: ${ }^{31}$

$\mathrm{BRDF}=f\left(\theta_{r}, \phi_{r} ; \theta_{i}, \phi_{i}\right)=\frac{\mathrm{d} L_{r}\left(\theta_{r}, \phi_{r} ; \theta_{i}, \phi_{i}\right)}{\mathrm{d} E_{i}\left(\theta_{i}, \phi_{i}\right)}$.

Harvey and Shack (1976) developed a linear systems formulation of surface scattering phenomena in which the scattering behavior is characterized by a surface transfer function. $^{\text {32,33 }}$ The Fourier transform of this surface transfer function yielded the scattered light distribution (also a scattered radiance function). This transfer function characterization of scattering surfaces was generalized in the 1980s to include grazing-incidence effects in x-ray telescopes, and mid-spatial-frequency surface errors that span the gap between figure and finish errors. ${ }^{34}$ This allowed accurate predictions of image degradation due to scattering effects from residual optical fabrication errors on NASA's Chandra Observatory and NOAA's Solar X-ray Imager (SXI). ${ }^{35,36}$

Surface scattering continues to be an important problem in diverse areas of science and engineering in the twentyfirst century. In 2004, Elfouhaily and Guerin stated that "Approximate models are still a necessity due to the insurmountable numerical complexity of realistic scattering problems. Even today's machines cannot cope with the enormous amount of computing demanded in the case of rigorous numerical calculations of the most general threedimensional electromagnetic wave scattering from dielectric or conducting multi-scale surfaces." 37 They went on to provide an exhaustive critical survey of approximate theories of the scattering of waves from random rough surfaces. They attempted to classify and characterize more than thirty different approximate methods. These were all variants of the small-perturbation (Rayleigh-Rice) method, the Kirchhoff approach, or the so-called unified methods, which tried to bridge the gap between the two. This exhaustive survey included 260 references: 177 published since 1980, 130 published since 1990, and 41 published between 2000 and 2004. They concluded that "there does not seem to be a universal method that is to be preferred systematically. All methods present a compromise between versatility, simplicity, numerical efficiency, accuracy and robustness, with a different weighting in these various fields." Elfouhaily and Guerin went on to state that "no approximate model has fulfilled all listed criteria. Moreover, most models did not even satisfy half of the requirements." Their final statement was: "There is still room for improvement in the development of approximate scattering methods."

One last note on the historical background of surface scattering theory: There have developed two distinct camps of researchers in that area. First, there are the theoretically inclined scientists, who are perhaps drawn to the investigation of surface scattering because it is an area of practical interest that is mathematically and theoretically challenging. Second, there are the researchers in computer vision and computer animation, who are less interested in rigorously solving the surface scattering problem. Their primary concern is having a surface scattering model that results in the rendering of realistic surfaces, textures, objects, and scenes under a wide variety of illumination conditions. This second group has evaluated, implemented, and referenced ${ }^{38}$ an empirically modified Beckmann-Kirchhoff model reported in a non-peer-reviewed conference proceedings in $1998 .^{39}$ This paper is intended to compare that modified Beckmann-Kirchhoff model quantitatively with both the Rayleigh-Rice and the classical Beckmann-Kirchhoff theories and to report it in the archival literature.

\section{Diffracted Radiance: New Insight into Nonparaxial Diffraction Phenomena}

An extension (and generalization) of the Harvey-Shack surface scattering theory has recently led to a linear systems formulation of nonparaxial scalar diffraction theory. ${ }^{40,41}$ The incorporation of the principles (and proper terminology $y^{31}$ ) of radiometry is crucial to the new insight and understanding obtained from this treatment of nonparaxial diffraction phenomena. We thus briefly review the definitions of a few radiometric quantities. In the past scientists have generally used the word intensity to mean the flow of energy per unit area per unit time. However, by international, if not universal, agreement, that term is slowly being replaced by the word irradiance: ${ }^{42}$

irradiance $\equiv E=\frac{\partial P}{\partial A_{c}} \quad$ (watts per unit area).

Irradiance is thus defined as the radiant power density incident on a collecting surface (hence the subscript $c$ ). $R a$ diant intensity, on the other hand, is power per unit solid angle radiated from a source (particularly a point source or source that has negligible area compared to the square of the viewing distance): ${ }^{43,44}$

radiant intensity $\equiv I=\frac{\partial P}{\partial \omega_{c}} \quad$ (watts per steradian)

And radiance, the radiometric analog to the more familiar photometric term brightness, is defined as radiant power per unit solid angle per unit projected source area. The quantity radiance is used to characterize an extended source, that is, one that has appreciable area compared to the square of the viewing distance. ${ }^{4,44}$ In differential form,

$$
\begin{aligned}
\text { radiance } \equiv & L=\frac{\partial^{2} P}{\partial \omega_{c} \partial A_{s} \cos \theta_{s}} \\
& \times(\text { watts per steradian per unit projected area })
\end{aligned}
$$

The radiance of a source is, in general, a function of position on the source and a function of the two angular variables $\theta_{s}$ and $\phi_{s}$ in conventional spherical coordinates.

In Ref. 40, diffracted radiance (not irradiance or intensity) was shown to be a fundamental quantity predicted by the Fourier transform of the optical disturbance emerging from a diffraction aperture, and a paraxial limitation was not necessary in this linear systems formulation of wideangle diffraction phenomena: 
$L^{\prime}\left(\alpha, \beta-\beta_{0}\right)= \begin{cases}K \frac{\lambda^{2}}{A_{s}}\left|\mathcal{F}\left\{U_{0}^{\prime}(\hat{x}, \hat{y} ; 0) \exp \left(i 2 \pi \beta_{0} \hat{y}\right)\right\}\right|^{2} & \text { for } \alpha^{2}+\beta^{2} \leqslant 1, \\ 0 & \text { for } \alpha^{2}+\beta^{2}>1 .\end{cases}$

Here $L^{\prime}$ is the classical radiance (radiant power per unit solid angle per unit projected area), $A_{s}$ is the source area (i.e., the area of the diffracting aperture), and $U_{0}^{\prime}$ is the complex amplitude distribution emerging from the diffracting aperture. Note that Ref. 40 utilized a scaled coordinate system in which the spatial variables are normalized by the wavelength of the light $(\hat{x}=x / \lambda, \hat{y}=y / \lambda)$. The reciprocal variables $\alpha$ and $\beta$ were then the direction cosines of the propagation vectors of the angular spectrum of plane waves discussed by Ratcliff, ${ }^{45}$ Goodman, ${ }^{46}$ and Gaskill. ${ }^{47}$ The renormalization constant $K$ in this reformulation of scalar diffraction theory is given by the following expression:

$K=\frac{\int_{\alpha=-\infty}^{\infty} \int_{\beta=-\infty}^{\infty} L\left(\alpha, \beta-\beta_{0}\right) \mathrm{d} \alpha \mathrm{d} \beta}{\int_{\alpha=-1}^{1} \int_{\beta=-\left(1-\alpha^{2}\right)^{1 / 2}}^{\left(1-\alpha^{2}\right)^{1 / 2}} L\left(\alpha, \beta-\beta_{0}\right) \mathrm{d} \alpha \mathrm{d} \beta}$,

and it only differs from unity if the diffracted radiance distribution function extends beyond the unit circle in direction cosine space (i.e., only if evanescent waves are produced). ${ }^{40}$ The well-known Wood's anomalies that occur in diffraction grating efficiency measurements are entirely consistent with this predicted renormalization in the presence of evanescent waves. ${ }^{48}$ This renormalization process is also consistent with the law of conservation of energy. However, it is significant that this linear systems formulation of nonparaxial scalar diffraction theory has been derived by the application of Parseval's theorem and not by merely heuristically imposing the law of conservation of energy.

Equations (5) and (6) have been applied to the special case of perfectly conducting sinusoidal phase gratings to predict a variety of wide-angle (nonparaxial) diffraction grating effects. ${ }^{49}$ These include: (i) the redistribution of energy from the evanescent orders to the propagating ones, (ii) the angular broadening (and apparent shifting) of wideangle diffracted orders, and (iii) nonparaxial diffraction efficiencies predicted with an accuracy usually thought to require rigorous electromagnetic vector theory. It is also shown in Ref. 49 that these scalar predictions agree with the behavior of transverse electric (TE, or $s$ ) polarized light, but not transverse magnetic (TM, or $p$ ) or unpolarized light.

Since random rough surfaces can be Fourier decomposed into a superposition of sinusoidal reflection gratings, the success with the sinusoidal phase grating has led to the current reexamination of the classical Beckmann-Kirchhoff scattering theory. Our empirically modified BeckmannKirchhoff model is here shown to exhibit (for TE, or $s$, polarization) the advantages of both the classical RayleighRice theory and the classical Beckmann-Kirchhoff theory, with the limitations of neither. In a later paper, we will report on our attempt to quasi-vectorize this scalar treatment.

\section{Nonintuitive Surface Scattering Measurements}

A detailed experimental investigation of light scattering from well-characterized random surfaces was reported by O'Donnell and Mendez in $1987 .{ }^{50}$ Several puzzling effects were observed when comparing experimental scattered intensity measurements with classical Beckmann-Kirchhoff scattering theory. The surfaces were made by exposing photoresist to a laser speckle pattern and then processing it and coating it with gold. The surface autocovariance (ACV) function was almost a perfect Gaussian. The measured rootmean-square (rms) surface roughness was $\sigma_{s}=2.27 \mu \mathrm{m}$, and the measured autocovariance length $\left(e^{-1}\right.$ half width of the ACV function) was $l_{c}=20.9 \mu \mathrm{m}$. For the small slopes represented by these parameters, multiple-scattering effects are negligible and conventional high-angular-resolution scattering data were measured for two wavelengths, $\lambda$ $=0.6328 \mu \mathrm{m}$ and $\lambda=10.6 \mu \mathrm{m}$, and two angles of incidence, $\theta_{i}=20 \mathrm{deg}$ and $\theta_{i}=70 \mathrm{deg}$. At these wavelengths and incident angles this surface is far too rough to satisfy the Rayleigh-Rice smooth-surface criterion.

For a wavelength of $10.6 \mu \mathrm{m}$ and modest angles of incidence the Beckmann-Kirchhoff solution agrees quite well with the experimental data; however, a persistent tendency for the data to be narrower than the theory was observed [see Fig. 1(a)]. This was nonintuitive in that experimental error sources (jitter, turbulence effects, etc.) would tend to make the experimental curve broader than the theoretical curve. There is no discernible specular beam for an incident angle of $20 \mathrm{deg}$, because the total integrated scatter (TIS) is almost unity: $:^{30,34}$

TIS $=1-\exp \left[-\left(4 \pi \cos \theta_{i} \sigma_{s} / \lambda\right)^{2}\right]$.

For a wavelength of $\lambda=10.6 \mu \mathrm{m}$ and an incident angle of $\theta_{i}=70$ deg there is a specular beam containing more than $40 \%$ of the reflected radiant power; however, the data points influenced by it have been omitted from the experimental data shown in Fig. 1(b). Note that the peak of the scattering function does not lie in the specular direction; instead, it lies approximately $10 \mathrm{deg}$ inside the specular beam. Likewise, the Beckmann-Kirchhoff theory no longer exhibits symmetry about the specular direction and shows a similar shift of the peak of the diffuse component of the scattered light distribution. The authors of Ref. 50 offer no explanation for this nonintuitive behavior.

Finally, Fig. 1(c) illustrates the data for a wavelength of $\lambda=0.6328 \mu \mathrm{m}$ and an angle of incidence of $\theta_{i}=70 \mathrm{deg}$. The experimental data are highly asymmetrical about the specular direction (note that there is no specular beam at this wavelength) and drop smoothly to zero at $-90 \mathrm{deg}$. The 

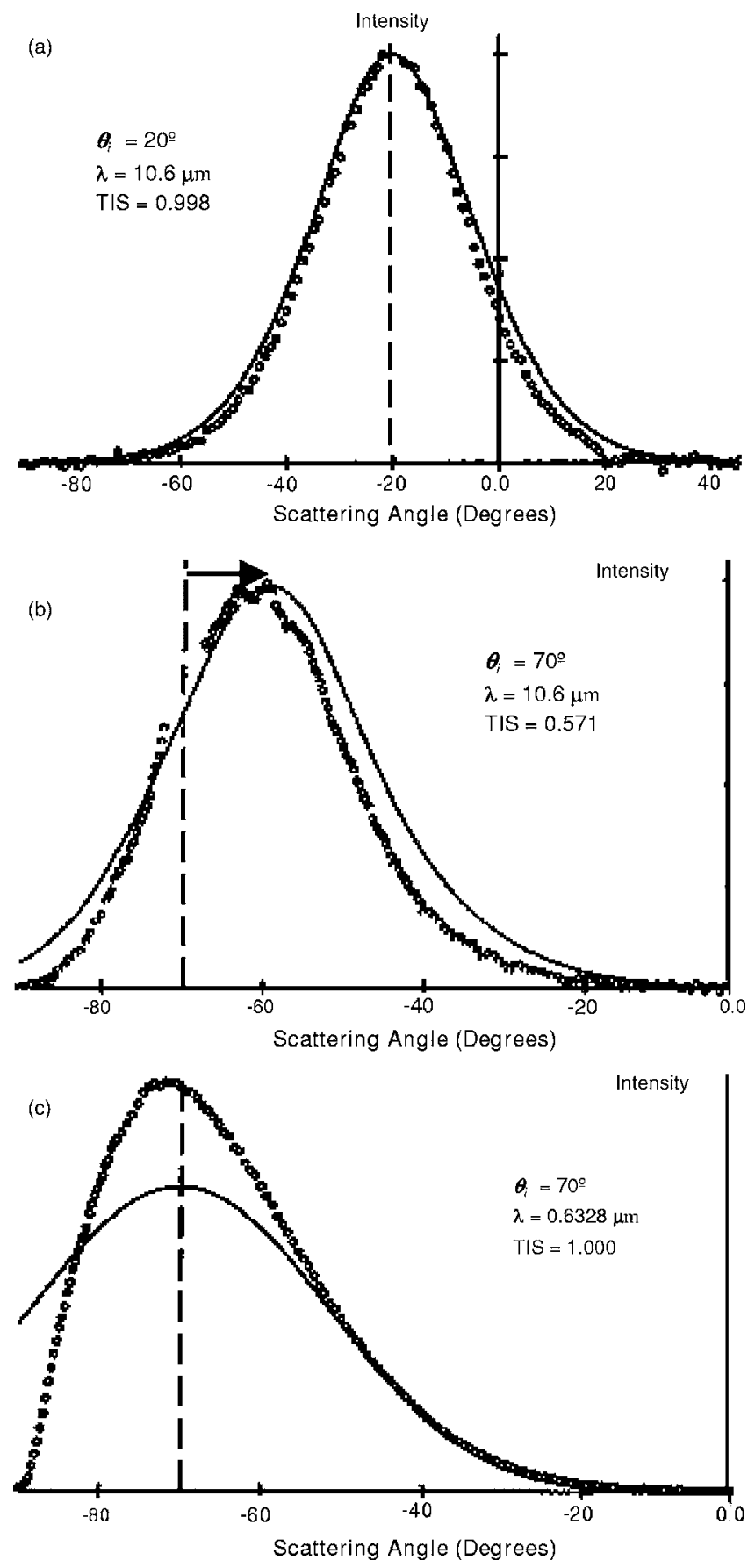

Fig. 1 Nonintuitive surface scattering effects. The solid lines represent classical Beckmann-Kirchhoff surface scattering theory; the circles indicate experimental measurements. (Reprinted with permission from the authors of Ref. 50).

Beckmann theory predicts a symmetrical intensity distribution about the specular direction, though it is unphysical in that it exhibits a discontinuity at $-90 \mathrm{deg}$. The authors of Ref. 50 suggest that the reason the Beckmann theory fails at this angle of incidence may be primarily shadowing and multiple scattering effects, and state that they are unaware of any available theory that compares to their measured data.

\section{Modified Beckmann-Kirchhoff Surface Scattering Model}

When applied to O'Donnell and Mendez's scattering surface, the Harvey-Shack surface scattering theory predicted a shift-invariant angle spread function (scattered radiance distribution) which, when truncated and renormalized in accordance with Eq. (5) and Eq. (6), then converted to radiant intensity by applying Lambert's cosine law, qualitatively and intuitively explains all three of the nonintuitive scattering effects illustrated in Fig. $1{ }^{40}$ These results, and the remarkable accuracy with which the nonparaxial scalar diffraction theory of Ref. 40 allowed us to calculate diffraction grating efficiencies in Ref. 49, have led us to believe that the nonintuitive surface scattering effects reported by O'Donnell and Mendez, and illustrated in Fig. 1, might be the result of inappropriately comparing different radiometric quantities.

Note that O'Donnell and Mendez have labeled the curves in Fig. 1 as scattered intensity versus scattering angle. Since the solid angle subtended by the collecting aperture of their scatterometer is constant as they scan the observation hemisphere, the voltage signal received from their instrument is indeed proportional to scattered intensity (W/sr). However, since diffracted radiance is the quantity predicted by the new nonparaxial scalar diffraction theory, we are led to question their interpretation that the Beckmann-Kirchhoff surface scattering theory predicts scattered intensity. The discontinuity in the solid curve (representing the Beckmann-Kirchhoff theory) at $-90 \mathrm{deg}$ in Fig. 1(b) and 1(c) is unphysical if the quantity being plotted is radiant intensity (W/sr); however, such a discontinuity would be allowed if plotting scattered radiance. (It should be pointed out that Beckmann and Spizzichino ${ }^{20}$ do not use conventional radiometric terminology.) O'Donnell and Mendez used Eq. (35) on p. 86 of Ref. 20, a closedform solution valid only for Gaussian surface autocovariance functions, which is written as

$D\{\rho\}=\frac{\pi l_{c}^{2} F^{2} \exp (-g)}{A_{s}} \sum_{m=1}^{\infty} \frac{g^{m}}{m ! m} \exp \left(\frac{v_{x y}^{2} l_{c}^{2}}{4 m}\right)$

where $A_{s}$ is the illuminated surface area; $l_{c}$ is the surface correlation length; $F$ is a geometrical factor defined as the following function of incident and scattered angles $(\theta$ and $\phi$ are the scattered angles in standard spherical coordinates):

$F=\frac{1+\left(\cos \theta_{i} \cos \theta-\sin \theta_{i} \sin \theta \cos \phi\right)}{\cos \theta_{i}\left(\cos \theta_{i}+\cos \theta\right)} ;$

$g$ is a measure of the phase variation introduced by an rms surface roughness of $\sigma_{s}$, given by

$g=\left[\frac{2 \pi \sigma_{s}}{\lambda}\left(\cos \theta_{i}+\cos \theta\right)\right]^{2}$

and

$v_{x y}=k\left(\sin ^{2} \theta_{i}-2 \sin \theta_{i} \sin \theta \cos \phi+\sin ^{2} \theta\right)^{1 / 2}$.

The quantity $D\{\rho\}$ in Eq. (8) is the time average of the squared modulus of the electric field vector and is called 


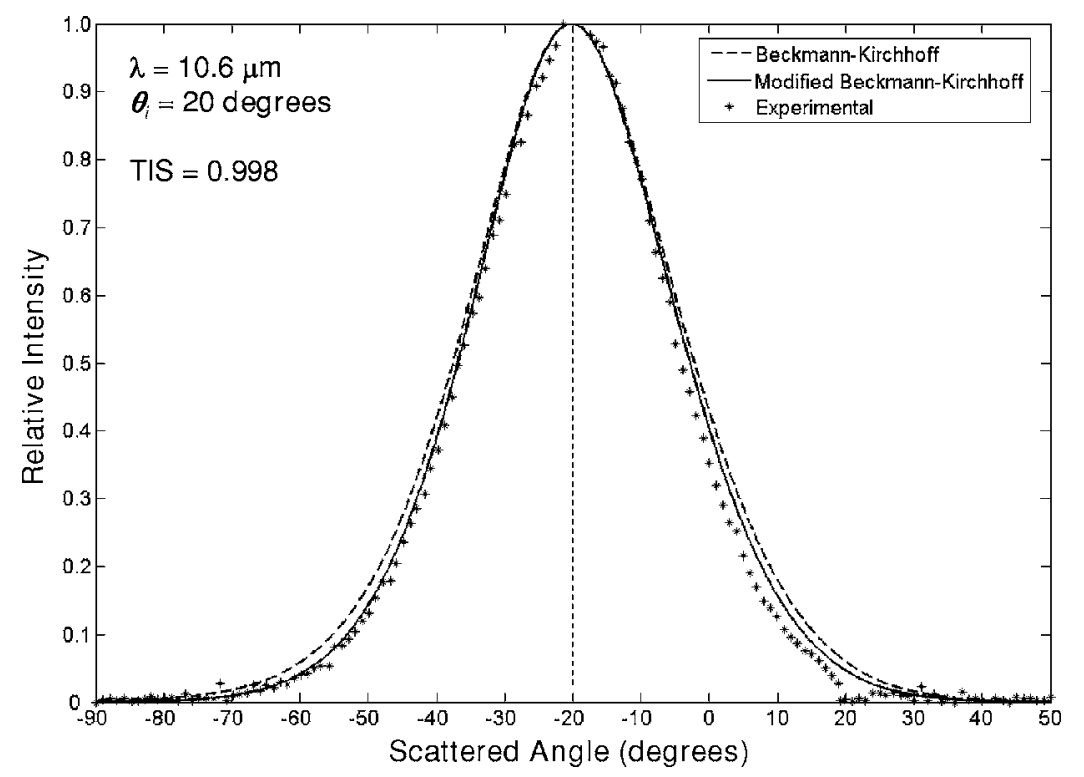

Fig. 2 Comparison of the classical Beckmann-Kirchhoff surface scattering theory, the modified Beckmann-Kirchhoff scattering model, and the O'Donnell-Mendez experimental data for a wavelength of $10.6 \mu \mathrm{m}$ and an incident angle of $20 \mathrm{deg}$.

the mean scattered power by Beckmann and Spizzichino. ${ }^{20}$ Most physicists would interpret this quantity to be proportional to radiant power density on the collecting surface (and perhaps call it intensity). The geometrical factor $F$ is perhaps an attempt to model the asymmetries that occur in measured scattered intensity profiles.

However, the new insight provided by Eq. (5) and Eq. (6) of this paper, in which diffracted radiance is presented as the fundamental quantity predicted by scalar diffraction theory, and the qualitative success of the Harvey-Shack surface scattering theory in explaining the nonintuitive behavior described, ${ }^{40}$ leads us to try an empirical modification of the Beckmann-Kirchhoff scattering theory. Three explicit empirical modifications include: (i) eliminating the geometrical factor $F^{2}$; (ii) introducing a renormalization factor, analogous to $K$ in Eq. (6), which takes account of the redistribution of radiant energy from the evanescent waves into the propagating waves; and (iii) equating the right side of Eq. (8) to the scattered radiance. We thus obtain the following modified Beckmann-Kirchhoff scattering model:

$L(\theta, \phi)=K \frac{\pi l_{c}^{2} \exp (-g)}{A_{s}} \sum_{m=1}^{\infty} \frac{g^{m}}{m ! m} \exp \left(\frac{v_{x y}^{2} l_{c}^{2}}{4 m}\right)$.

To obtain the scattered intensity distribution, we merely multiply the radiance by $\cos \theta$ and integrate over the illuminated area of the scattering surface: ${ }^{40,49}$

$I(\theta, \phi)=\int_{A_{s}} L(\theta, \phi) \cos \theta \mathrm{d} A_{s}=L(\theta, \phi) A_{s} \cos \theta$,

or

$I(\theta, \phi)=K \pi l_{c}^{2} \cos \theta \exp (-g) \sum_{m=1}^{\infty} \frac{g^{m}}{m ! m} \exp \left(\frac{v_{x y}^{2} l_{c}^{2}}{4 m}\right)$.
Recall that for a wavelength of $10.6 \mathrm{~mm}$ and an incident angle of $20 \mathrm{deg}$, almost all of the reflected light is scattered (TIS $=0.998)$ and there is virtually no specular beam. In Fig. 2, scattered intensity predictions from this new, modified Beckmann-Kirchhoff model expressed by Eq. (14) are superimposed on the previous curves of Fig. 1(a). Since we only have one-dimensional experimental scattering profile data, we merely normalize the peak of the scattering function to unity, and label the curve "relative intensity." For this modest 20-deg incident angle, the departures of the predictions from the classical Beckmann-Kirchhoff and the modified Beckmann-Kirchhoff surface scattering model are not severe; however, the modified model does match the experimental data more closely.

In Fig. 3 the scattered intensity predictions from the new modified Beckmann-Kirchhoff model are superimposed on the previous curves in Fig. 1(b). Recall that for a 70-deg incident angle and a wavelength of $10.6 \mu \mathrm{m}$ the departure between the experimental data and the predictions of the classical Beckmann-Kirchhoff theory was significant, and there was a particularly bothersome (unphysical) discontinuity in the predicted curve at a scattering angle of $-90 \mathrm{deg}$. At this large incident angle, almost half of the reflected radiant power resides in the specular beam (TIS $=0.571$, and, as previously noted, the peak of the scattering function is shifted approximately $10 \mathrm{deg}$ from the specular direction. The modified Beckmann-Kirchhoff model once again more closely matches the experimental measurements, and it does not exhibit the unphysical discontinuity at a scattering angle of $-90 \mathrm{deg}$.

For the much shorter wavelength of $\lambda=0.6328 \mu \mathrm{m}$, O'Donnell and Mendez's surface must be categorized as very rough $\left(\sigma_{s} / \lambda=3.59\right)$, and clearly, for such a rough surface all the incident light is scattered (no specular beam). 


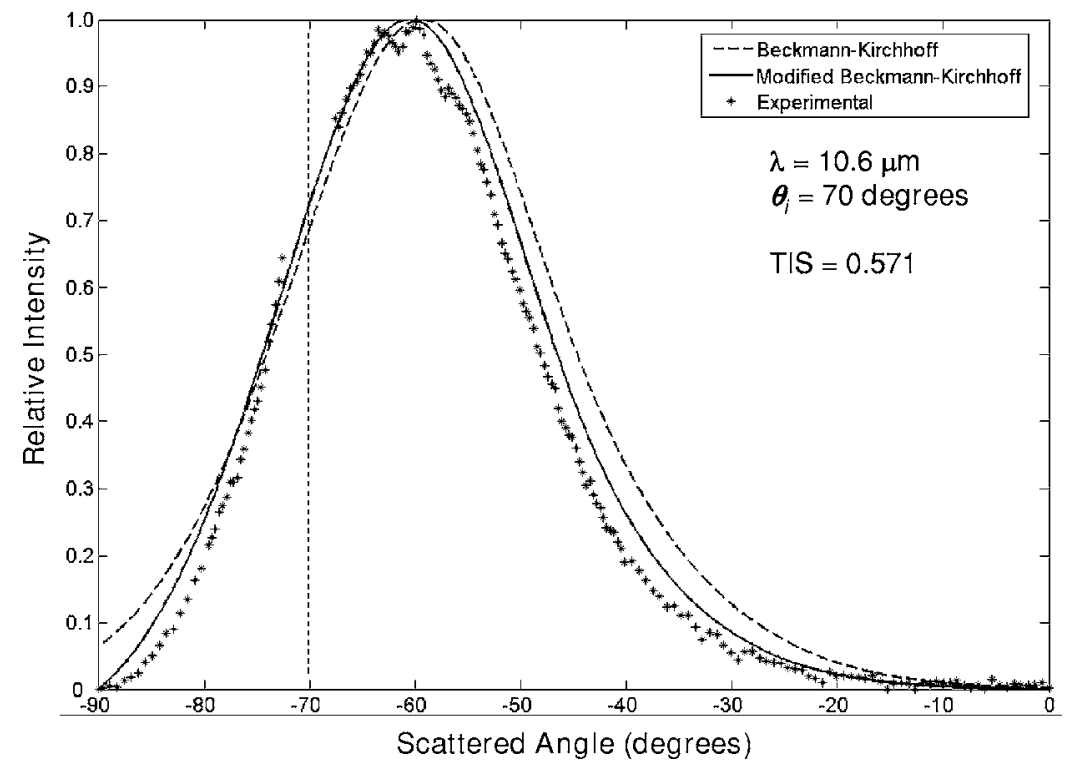

Fig. 3 Comparison of the classical Beckmann-Kirchhoff surface scattering theory, the modified Beckmann-Kirchhoff scattering model, and the O'Donnell-Mendez experimental data for a wavelength of $10.6 \mu \mathrm{m}$ and an incident angle of 70 deg.

Equations (12) and (14) do not converge; hence, we use the classical Beckmann-Kirchhoff equation [Eq. (48) on p. 87 of Ref. 20]:

$D\{\rho\}=\frac{\pi l_{c}^{2} F^{2}}{A_{s} v_{z}^{2} \sigma_{s}^{2}} \exp \left(\frac{v_{x y}^{2} l_{c}^{2}}{4 v_{z}^{2} \sigma_{s}^{2}}\right)$,

where

$v_{z}=-k\left(\cos \theta_{i}+\cos \theta\right)$.

As before, we make an empirical modification by eliminating the geometrical factor $F^{2}$, adding the renormalization constant $K$, and equating the right side of Eq. (15) to the scattered radiance, thus obtaining the following modified Beckmann-Kirchhoff scattering model for very rough surfaces:

$L(\theta, \phi)=K \frac{\pi l_{c}^{2}}{A v_{z}^{2} \sigma_{s}^{2}} \exp \left(-\frac{v_{x y}^{2} l_{c}^{2}}{4 v_{z}^{2} \sigma_{s}^{2}}\right)$.

Again converting radiance to intensity, we obtain

$I(\theta, \phi)=K \frac{\pi l_{c}^{2} \cos \theta}{v_{z}^{2} \sigma_{s}^{2}} \exp \left(-\frac{v_{x y}^{2} l_{c}^{2}}{4 v_{z}^{2} \sigma_{s}^{2}}\right)$.

In Fig. 4, scattered intensity predictions from the modified Beckmann-Kirchhoff model expressed by Eq. (14) are superimposed on the previous curves in Fig. 1(c). Again we normalize the peak of the modified Beckmann-Kirchhoff scattering profile to unity. For this short wavelength and a 70-deg incident angle, the departure between the predictions of the classical and the modified theory is quite dramatic. The classical Beckmann-Kirchhoff theory indicates a very significant (unphysical) discontinuity at a scattering angle of -90 deg and is clearly not capable of making accurate surface scattering predictions for very rough surfaces at these large incident angles; however, the empirically modified Beckmann-Kirchhoff model agrees extremely well with the experimental data.

\section{Rayleigh-Rice Vector Perturbation Surface Scattering Theory}

Using Stover's notation for the Rayleigh-Rice vector perturbation surface scattering theory, ${ }^{30}$ the normalized (by the incident radiant power) scattered intensity in a given differential solid angle can be written as

$\frac{\left(\mathrm{d} P / \mathrm{d} \Omega_{s}\right) \mathrm{d} \Omega_{s}}{P_{i}}=\frac{16 \pi^{2}}{\lambda^{4}} \cos \theta_{i} \cos ^{2} \theta Q S\left(f_{x}, f_{y}\right) \mathrm{d} \Omega_{s}$.

The dimensionless quantity $Q$ is the polarization-dependent reflectance of the surface. For an $s$-polarized source, and measurements made in the plane of incidence, $Q$ is given exactly by the geometric mean of the sample specular reflectances at $\theta_{i}$ and $\theta$ :

$Q=\left[R_{S}\left(\theta_{i}\right) R_{s}(\theta)\right]^{1 / 2}$.

For highly reflective surfaces this means that $Q$ is very nearly equal to a constant for all scattering angles. In Eq. (19), $S\left(f_{\mathrm{x}}, f_{\mathrm{y}}\right)$ is the two-sided, two-dimensional surface power spectral density (PSD) function expressed in terms of the sample spatial frequencies

$f_{x}=\frac{\sin \theta \cos \phi-\sin \theta_{i}}{\lambda}$ and $f_{y}=\frac{\sin \theta \sin \phi}{\lambda}$.

This Rayleigh-Rice surface scattering theory is widely accepted and has been extensively validated even for large scattered and incident angles for smooth surfaces $\left(\sigma_{s} / \lambda\right.$ $\ll 1)$. We thus compare the modified Beckmann-Kirchhoff 


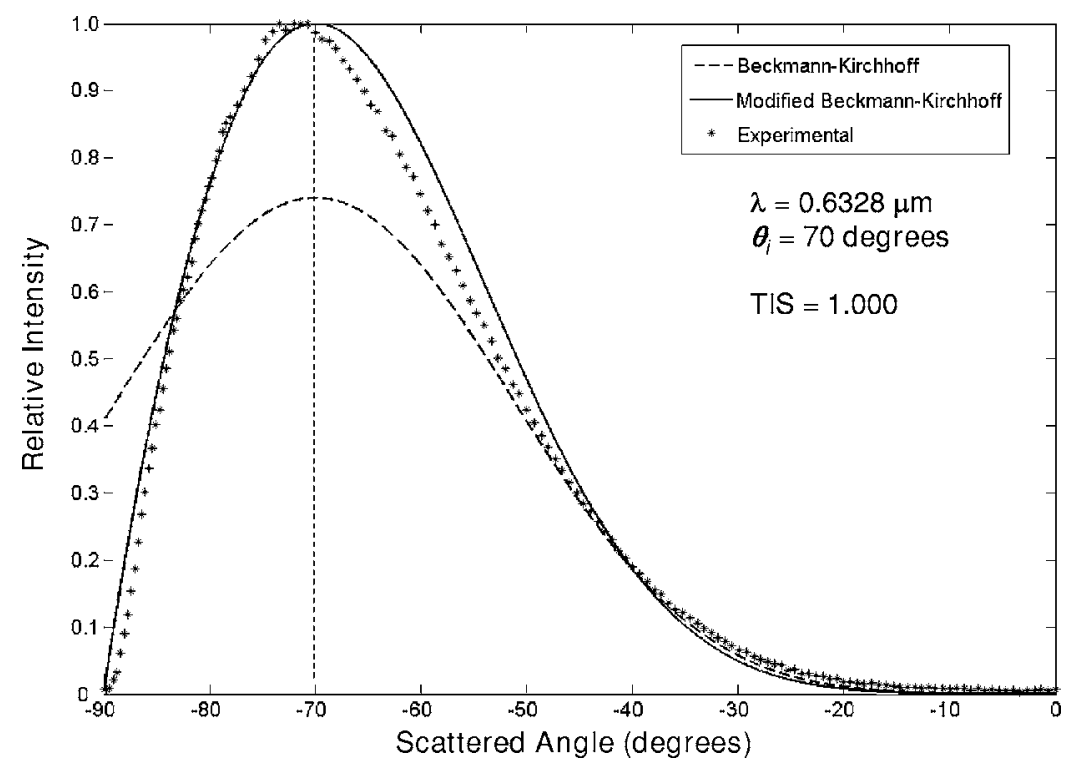

Fig. 4 Comparison of the classical Beckmann-Kirchhoff surface scattering theory, the modified Beckmann-Kirchhoff scattering model, and the O'Donnell-Mendez experimental data for a wavelength of $0.6328 \mu \mathrm{m}$ and an incident angle of $70 \mathrm{deg}$.

surface scattering model with predictions from the Rayleigh-Rice theory. Assuming a Gaussian autocovariance (ACV) function, we can write

$$
\mathrm{ACV}=\sigma_{s}^{2} \exp \left[-\left(r / l_{c}\right)^{2}\right]
$$

The surface PSD is given by the Fourier transform of the ACV function; hence, the $x$ profile is given by

$S\left(f_{x}\right)=\pi \sigma_{s}^{2} l_{c}^{2} \exp \left[-\left(\frac{\sin \theta-\sin \theta_{i}}{\lambda / \pi l_{c}}\right)^{2}\right]$.

Figure 5 illustrates that for smooth surfaces $(\sigma / \lambda$
$=0.02)$ with normal incidence and a surface autocovariance width $\left(l_{c} / \lambda=1.2\right)$ that results in modest scattering angles, all three of the scattering theories yield virtually identical results.

If we keep the same rms surface roughness, but shorten the surface autocovariance length by a factor of $3\left(l_{c} / \lambda\right.$ $=0.4$ ), the scattering angles become quite large, and the classical Beckmann-Kirchhoff theory starts to break down, as evidenced by the unphysical discontinuity in the predicted scattered intensity at scattering angles of $\pm 90 \mathrm{deg}$,

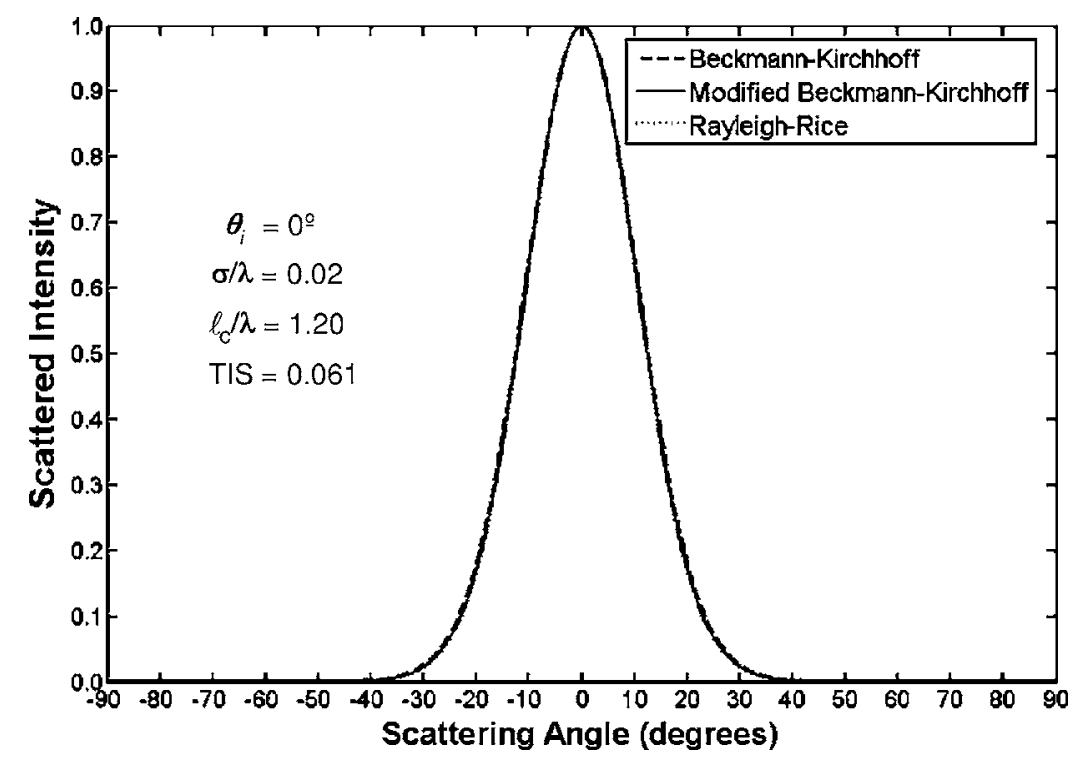

Fig. 5 For a smooth surface $(\sigma / \lambda=0.02)$ with small incident and scattering angles, the classical Beckmann-Kirchhoff theory, modified Beckmann-Kirchhoff model, and Rayleigh-Rice surface scattering theory all yield virtually identical results. 


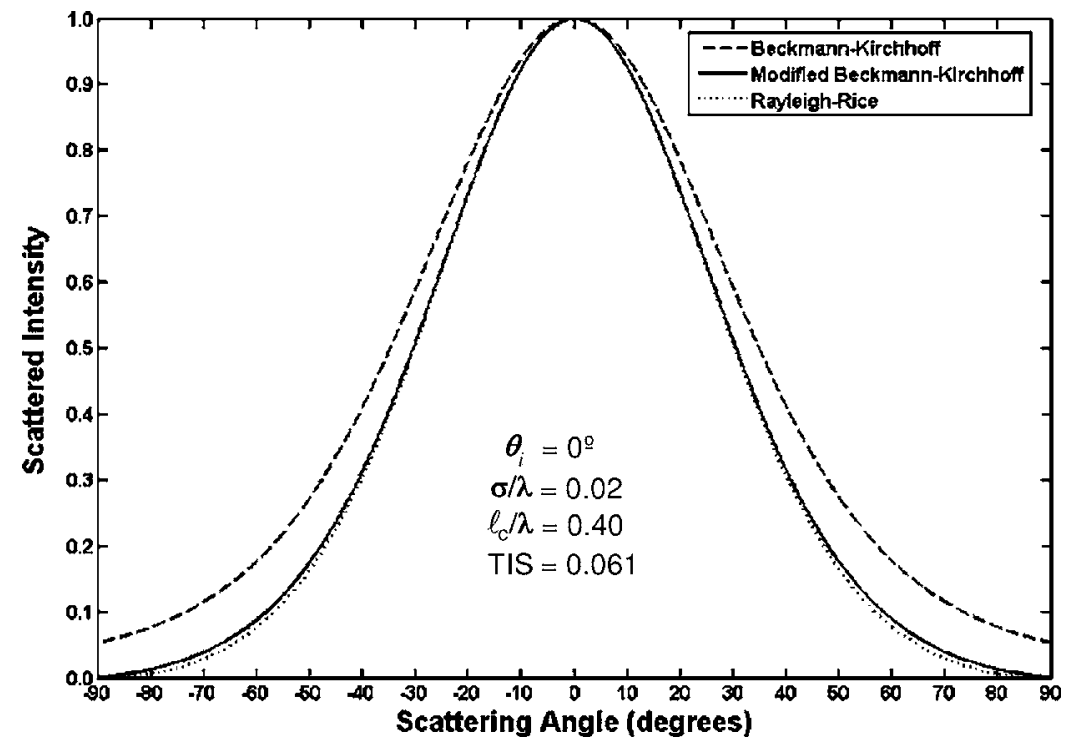

Fig. 6 For a smooth surface with large scattering angles, the classical Beckmann-Kirchhoff theory starts to break down; however, the modified Beckmann-Kirchhoff model continues to agree extremely well with the Rayleigh -Rice surface scattering theory.

shown in Fig. 6. The modified Beckmann-Kirchhoff model continues to agree extremely well with the Rayleigh-Rice surface scattering theory.

Finally, if we maintain the smooth-surface and narrowsurface autocovariance length producing the wide scattering angles, and introduce a 45-deg incident angle, the empirically modified Beckmann-Kirchhoff model continues to agree remarkably well with the well-established RayleighRice theory; however, the classical Beckmann-Kirchhoff theory exhibits markedly different behavior, as shown in Fig. 7.

\section{Summary and Conclusions}

Rayleigh-Rice ${ }^{6}$ or classical Beckmann-Kirchhoff ${ }^{20}$ theories are commonly used to predict surface scattering behavior. The Rayleigh-Rice vector perturbation theory agrees well with experimental wide-angle scattering measurements from smooth $\left(\sigma_{s} / \lambda \ll 1\right)$ surfaces for arbitrary incident and scattering angles. The classical Beckmann-Kirchhoff scattering theory is valid for rougher surfaces, but contains a paraxial (small-angle) assumption that limits its ability to accurately handle wide-angle scattering and large angles of

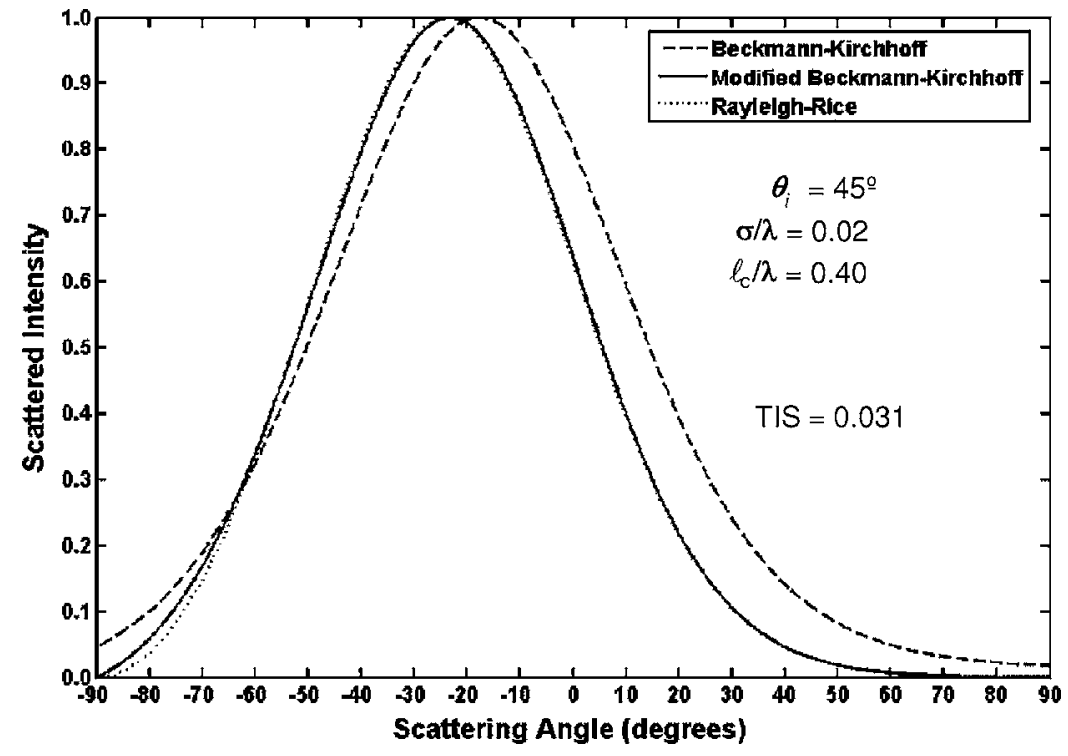

Fig. 7 For smooth surfaces with large scattering and incident angles, the modified BeckmannKirchhoff model continues its excellent agreement with the Rayleigh-Rice theory. The classical Beckmann-Kirchhoff theory exhibits markedly different behavior. 
incidence. The closed-form equations provided by Beckmann are also limited to scattering surfaces with Gaussian autocovariance functions. ${ }^{20} \mathrm{~A}$ recently developed nonparaxial scalar diffraction theory ${ }^{40}$ has led to an empirical modification of the classical Beckmann-Kirchhoff surface scattering theory that agrees extremely well with experimental data for rough surfaces, even for large incident and scattered angles. This modified Beckmann-Kirchhoff model also exhibits excellent agreement with the well-established Rayleigh-Rice vector perturbation surface scattering theory (for TE, or $s$, polarization) within its domain of applicability for smooth surfaces (comparisons performed for surfaces with Gaussian autocovariance functions).

Current research is resulting in the theoretical development of a linear systems formulation of surface scattering theory (generalized Harvey-Shack theory) for arbitrary surface statistics that will reduce to the empirically modified Beckmann-Kirchhoff model for surfaces with a Gaussian autocovariance function. A future paper will report on this development and compare its predictions with available experimental scattering data from non-Gaussian surfaces.

\section{References}

1. Lord Rayleigh, The Theory of Sound, Vol. II, Macmillan \& Company (1986).

2. Lord Rayleigh, "Polish," Nature (London) 64, 385-388 (1901).

3. Lord Rayleigh, "On the dynamical theory of gratings," Proc. R. Soc. London, Ser. A A79, 399-416 (1907).

4. T. K. Chenmoganadam, "On the specular reflection from rough surfaces," Phys. Rev. 13, 96-101 (1919).

5. U. Fano, "The theory of anomalous diffraction gratings and of quasistationary waves on metallic surfaces (Sommerfeld's waves)," J. Opt. Soc. Am. 31, 213-222 (1941).

6. S. O. Rice, "Reflection of electromagnetic waves from slightly rough surfaces," Commun. Pure Appl. Math. 4, 351 (1951).

7. C. I. Beard, "Coherent and incoherent scattering of microwaves from the ocean," IRE Trans. Antennas Propag. AP-9, 470-483 (1961).

8. I. M. Fuks, "Theory of radio wave scattering at a rough sea surface," Sov. Radiophys. 9, 513-519 (1966).

9. F. G. Bass, "Very high frequency radiowave scattering by a disturbed sea surface," IRE Trans. Antennas Propag. AP-16, 554-568 (1968).

10. D. E. Barrick, "Rough surfaces," in Radar Cross Section Handbook, Vol. 2, G. T. Ruck et al., Eds., pp. 671-772, Plenum Press, New York (1970).

11. F. B. Daniels, "A theory of radar reflection from the moon and planets," J. Geophys. Res. 66, 1781-1788 (1961).

12. J. V. Evans and G. H. Pettengill, "The scattering behavior of the moon at wavelengths of 3.6, 68, and 784 centimeters," J. Geophys. Res. 68, 423-447 (1963).

13. A. K. Fung and R. K. Moore, "Effects of structure size on moon and earth radar returns at various angles," J. Geophys. Res. 69, 1075-1081 (1964)

14. T. Hagafors, "Backscattering from an undulating surface with applications to radar returns from the moon," J. Geophys. Res. 69, 3779-3784 (1964)

15. L. M. Brekhovskikh, "Difrakcya voln na nerovnoj poverhnosti: 1, Obschaya teoriya," Zh. Eksp. Teor. Fiz. 23, 275-288 (1952).

16. L. M. Brekhovskikh, "Difrakcya voln na nerovnoj poverhnosti: 2, Prilozheniya Obschej Teorii," Zh. Eksp. Teor. Fiz. 23, 289-404 (1952).

17. M. A. Isakovich, "Wave scattering from a statistically rough surface," Zh. Eksp. Teor. Fiz. 23, 305-314 (1952).

18. C. Eckart, "The scattering of sound from the sea surface," J. Acoust. Soc. Am. 25, 566-570 (1953).

19. H. Davies, "The reflection of electromagnetic waves from a rough surface," Proc. Inst. Electr. Eng. 101, 209-214 (1954).

20. P. Beckmann and A. Spizzichino, The Scattering of Electromagnetic Waves from Rough Surfaces, Pergamon Press, New York (1963).

21. E. L. Church and J. M. Zavada, "Residual surface roughness of diamond-turned optics," Appl. Opt. 14, 1788-1795 (1975).

22. E. L. Church, H. A. Jenkinson, and J. M. Zavada, "Measurement of the finish of diamond-turned metal surfaces by differential scattering," Opt. Eng. 16, 360-374 (1977).

23. E. L. Church, H. A. Jenkinson, and J. M. Zavada, "Relationship between surface scattering and microtopographic features," Opt. Eng. 18, 125-136 (1979).
24. E. L. Church, "Fractal surface finish," Appl. Opt. 27, 1518-1526 (1988).

25. E. L. Church, P. Z. Takacs, and T. A. Leonard, "The prediction of BRDF's from surface profile measurements," Proc. SPIE $\mathbf{7 4 9}$, 136-150 (1989).

26. J. M. Elson and J. M. Bennett, "Vector scattering theory," Opt. Eng. 18, 116-124 (1979).

27. J. M. Bennett, "Measurement of the rms roughness, autocovariance function and other statistical properties of optical surfaces using a FECO scanning interferometer," Appl. Opt. 15, 2705-2721 (1976).

28. J. M. Bennett and L. Mattsson, Introduction to Surface Roughness and Scattering, Opt. Soc. of Am., Washington, D.C. (1989).

29. J. C. Stover, "Roughness characterization of smooth machined surfaces by light scattering," Appl. Opt. 14, 1796-1802 (1975).

30. J. C. Stover, Optical Scattering, Measurement and Analysis, 2nd ed., SPIE Press, Bellingham, WA (1995).

31. F. E. Nicodemus, "Reflectance nomenclature and directional reflectance and emissivity," Appl. Opt. 9, 1474-1475 (1970).

32. J. E. Harvey, "Light-scattering characteristics of optical surfaces," PhD Dissertation, Univ. of Arizona (1976).

33. J. E. Harvey, "Surface scatter phenomena: a linear, shift-invariant process," in Scatter from Optical Components, J. C. Stover, Ed., Proc. SPIE 1165, 87-99 (1989).

34. J. E. Harvey, E. C. Moran, and W. P. Zmek, "Transfer function characterization of grazing incidence optical systems," Appl. Opt. 27, 1527-1533 (Apr. 1988).

35. P. Glenn, P. Reid, A. Slomba, and L. P. Van Speybroeck, "Performance prediction of AXAF Technology mirror assembly using measured mirror surface errors," Appl. Opt. 27, 1539-1543 (1988).

36. J. E. Harvey and P. L. Thompson, "Generalized Wolter type I design for the Solar X-ray Imager (SXI)," Proc. SPIE 3766-14, 173-183 (1999).

37. T. M. Elfouhaily and C. A. Guerin, "A critical survey of approximate scattering wave theories from random rough surfaces," Waves Random Media 14, R1-R40 (2004).

38. H. Ragheb and E. R. Hancock, "Testing new variants of the Beckmann-Kirchhoff model against radiance data," Comput. Vis. Image Underst. 102(2), 145-168 (2006).

39. C. L. Vernold and J. E. Harvey, "A modified Beckmann-Kirchhoff scattering theory," in Proc. Int. Symp. on Optical Science, Engineering, and Instrumentation, Proc. SPIE 3426-05, (1998).

40. J. E. Harvey, C. L. Vernold, A. Krywonos, and P. L. Thompson, "Diffracted radiance: a fundamental quantity in a non-paraxial scalar diffraction theory," Appl. Opt. 38, 6469-6481 (Nov. 1999).

41. J. E. Harvey, C. L. Vernold, A. Krywonos, and P. L. Thompson, "Diffracted radiance: a fundamental quantity in a non-paraxial scalar diffraction theory: errata," Appl. Opt. 39, 6374-6375 (Dec. 2000).

42. J. M. Palmer, "Getting intense about intensity," Opt. Photonics News, p. 76 (Feb. 1995).

43. R. W. Boyd, Radiometry and the Detection of Optical Radiation, John Wiley \& Sons, New York (1983).

44. E. L. Dereniak and G. D. Boreman, Infrared Detectors and Systems, John Wiley \& Sons, New York (1996).

45. J. A. Ratcliff, "Some aspects of diffraction theory and their application to the ionosphere," in Reports of Progress in Physics, Vol. XIX A. C. Strickland, Ed., The Physical Society, London (1956).

46. J. W. Goodman, Introduction to Fourier Optics, 2nd ed., McGrawHill, New York (1996).

47. J. D. Gaskill, Linear Systems, Fourier Transforms, and Optics, Wiley, New York (1978).

48. R. W. Wood, "On a remarkable case of uneven distribution of light in a diffraction grating spectrum," Philos. Mag. 4, 396-410 (1902).

49. J. E. Harvey, A. Krywonos, and Dijana Bogunovic, "Non-paraxial scalar treatment of sinusoidal phase gratings," J. Opt. Soc. Am. A 23, 858-865 (2006).

50. K. A. O'Donnell and E. R. Mendez, "Experimental study of scattering from characterized random surfaces," J. Opt. Soc. Am. A 4, 1194-1205 (1987).

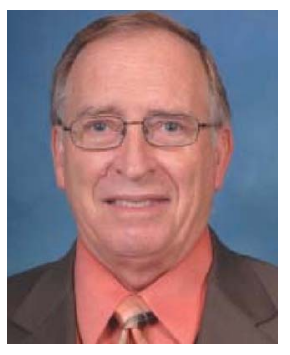

James E. Harvey received his $\mathrm{PhD}$ from the University of Arizona in 1976 . He is currently an associate professor in the College of Optics and Photonics at the University of Central Florida and a Senior Staff Member at the Center for Research and Education in Optics and Lasers (CREOL). Dr. Harvey is credited with more than 150 publications and conference presentations in the areas of diffraction theory, surface scattering phenomena, adaptive optics, wavefront sensing, beam sampling technology, optical properties of materials, and 
X-ray-EUV imaging systems. He is a member of the OSA and a fellow of SPIE.

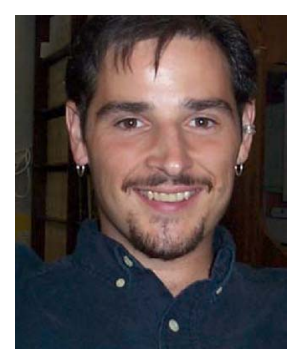

Andrey Krywonos received a bachelor's degree in photonics from the SUNY Institute of Technology at Utica, NY, in 1995, and a $\mathrm{PhD}$ degree in optics from the University of Central Florida in 2006. He is currently working with the Florida Space Institute (FSI) at the University of Central Florida in the area of optical design and analysis of space-based imaging spectrometers.
Cynthia L. Vernold received her BS degree in optics from the University of Rochester in 1987. From 1987 to 1993 she was an optical engineer for Perkin-Elmer Corp. and Hughes Danbury Optical Systems, Inc., where she worked on optical fabrication and metrologyrelated programs and managed their surface microtopography laboratory. After receiving a $\mathrm{PhD}$ degree in optical sciences from the University of Arizona in 1998, she worked briefly for the USAF Phillips Laboratory before founding and serving as president of Optical Insights, LLC, until its acquisition by Photometrics in 2005. 